\title{
Measuring Population Distributions and Catalytic Hierarchy of the Active Species in Gold on Metal Oxide Catalysts for Low Temperature CO Oxidation
}

\author{
Q. He ${ }^{1,2}$, S. Freakley ${ }^{3}$, J.K. Edwards ${ }^{3}$, G.J. Hutchings ${ }^{3}$ and C.J. Kiely ${ }^{1,3}$ \\ ${ }^{1}$ Departments of Materials Science and Chemical Engineering, Lehigh University, Bethlehem, PA \\ 18015, USA \\ ${ }^{2}$ Center for Nanophase Materials Sciences, Oak Ridge National Laboratory, Oak Ridge, Tennessee \\ 37831, USA \\ ${ }^{3}$ Cardiff Catalysis Institute, Cardiff University, Cardiff, CF10 3AT, UK
}

Supported gold on metal oxide $\left(\mathrm{MO}_{\mathrm{x}}\right)$ catalysts have been investigated extensively by many groups over recent years for their activity in low temperature CO oxidation. Materials prepared by co-precipitation, deposition precipitation and sol-immobilization methods have all been studied and found to display varying levels of activity for this reaction. Furthermore, such materials when examined using high resolution TEM and aberration corrected scanning transmission electron microscopy show considerable variations in their nanostructures [1]. So far, Au nano-particles [2,3], monolayer \& bilayer sub-nm $\mathrm{Au}$ clusters [4,5], extended Au rafts [6] and even highly dispersed Au atoms [7] have been identified in such catalysts and implicated as potential active species in the low temperature $\mathrm{CO}$ oxidation reaction (see Figure 1). The relative populations of the various supported $\mathrm{Au}$ species present in such $\mathrm{Au} / \mathrm{MO}_{\mathrm{x}}$ catalysts depends critically on the preparation method and precise synthesis conditions used. Naturally this structural complexity and catalytic variability has led to considerable debate over the nature of the active species/sites in such $\mathrm{Au} / \mathrm{MO}_{\mathrm{x}}$ catalysts.

It is likely that most of these supported $\mathrm{Au}$ species exhibit some catalytic activity for $\mathrm{CO}$ oxidation activity, albeit to varying degrees. In order to understand the overall activity exhibited by a particular catalyst sample containing a mixture of these supported Au species, one therefore has to gain some quantitative measure of their relative population distributions and an understanding of their ranking in terms of catalytic activity. It has been postulated that the overall activity can then be represented as a sum of the activity of each species present weighted by its relative abundance in the catalyst sample.

Although many catalysis papers nowadays present micrographs of supported Au catalysts showing a mixture of atoms, clusters and nanoparticles, their relative populations have at best been compared only in very qualitative terms. None have collected or analyzed this species population information in a statistically robust manner that allows proper correlations with catalytic measurements. Hence, we will present a reliable protocol that allows one to generate particle size distributions that include all species from atomic $\mathrm{Au}$ to $10 \mathrm{~nm}$ nanoparticles in real catalyst systems. Furthermore, we show that the data needs to be collected in terms of number density rather than number frequency measurements, which can subsequently be converted to mass fractions, surface atom fractions or periphery atom fractions for useful comparison with catalytic measurements. We will also discuss the advantages and practical limitations of our new particle size distribution measurement protocol.

We present an application of this new particle counting approach to study some systematic sets of $\mathrm{Au} / \mathrm{FeO}_{\mathrm{x}}$ materials that were specifically designed for use as low temperature $\mathrm{CO}$ oxidation catalysts. The quantitative knowledge of $\mathrm{Au}$ species populations obtained from appropriate analysis of HAADFSTEM images is used to rationalize some dramatic activity variations displayed by $\mathrm{Au} / \mathrm{FeO}_{\mathrm{x}}$ catalysts as a function of the calcination treatment they have received. In particular, we compare $\mathrm{Au} / \mathrm{FeO}_{\mathrm{x}}$ materials prepared via co-precipitation and deposition precipitation methods that exhibit diverging catalytic behaviors after heat treatment in air. After ruling out any catalyst support effects, and by correlating population densities with catalytic activity measurements, we are able to conclusively prove by 
experiment that a hierarchy of catalytic activities must exist for these different sized $\mathrm{Au}$ species on $\mathrm{FeO}$ In addition, we are able to affirm the hypothesis that the overall activity of the $\mathrm{Au} / \mathrm{FeO}_{\mathrm{x}}$ catalyst system for $\mathrm{CO}$ oxidation is the result of a complex population versus activity hierarchy relationship and that it is the weighted sum of the activity of each different species present on the catalytic surface. Furthermore, for the deposition precipitated materials, by making the reasonable assumption that the sub-nm Au clusters and 1-3 nm Au particles are the most active species, we use our experimental measurements of population distributions and catalytic activity to derive the intrinsic activities of the sub-nm clusters and 1-3 nm particles.

\section{References:}

[1] M. Haruta, Faraday Discuss., 152, (2011) 11.

[2] N. Lopez, J. Catal., 223, (2004) 232.

[3] Y. Liu, C.J. Jia, J. Yamasaki, O. Terasaki \& F. Schüth, Angew.Chem.Int.Ed., 49, (2010) 5771.

[4] A.A. Herzing, C.J. Kiely, A.F. Carley, P. Landon \& G.J. Hutchings, Science, 321, (2008) 1331.

[5] U. Landman, B. Yoon, C. Zhang, U. Heiz \& M. Arenz, Topics. Catal., 44, (2007) 145.

[6] M. Valden, X. Lai, \& D.W. Goodman, Science, 281, (1998) 1647.

[7] B. Qiao, J-X. Liang, A.Wang, C-Q. Xu, J Li, T. Zhang \& J. Liu, Nano Res., 8, (2015) 2913.

[8] The authors acknowledge funding from the National Science Foundation Major Research

Instrumentation program (GR\# MRI/DMR-1040229). Prof. M. Haruta is thanked for his many useful discussions and contributions to this work.

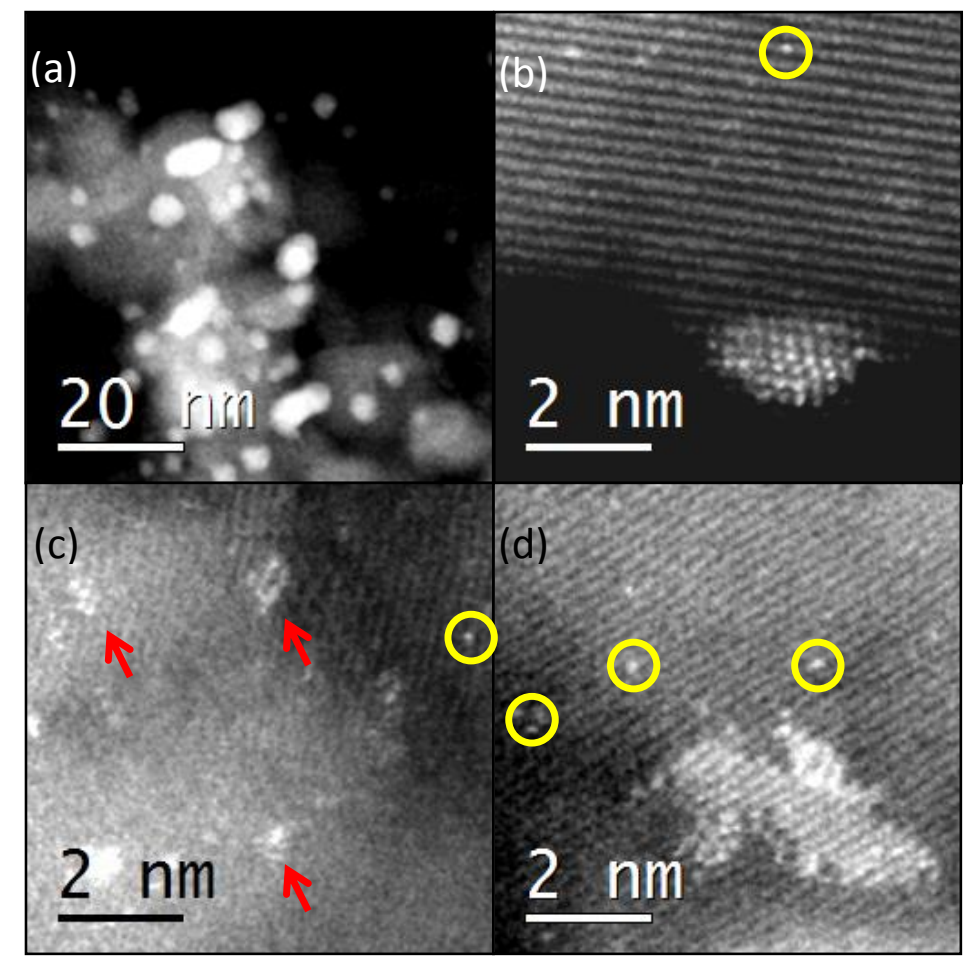

Figure 1. HAADF-STEM images of a co-precipitated $\mathrm{Au} / \mathrm{ZnO}$ low temperature $\mathrm{CO}$ oxidation catalyst showing the co-existence of supported Au nanoparticles (in (a) \& (b)), sub-nm Au clusters (arrowed in (c)), extended Au rafts (in d) and isolated Au atoms (circled in ((b), (c) \& (d)). 